\title{
Toenail Onychomycosis in a Portuguese Geriatric Population
}

\author{
N. Dias $\cdot$ C. Santos $\cdot$ M. Portela $\cdot$ N. Lima
}

Received: 7 March 2010/Accepted: 12 February 2011/Published online: 2 March 2011

(C) Springer Science+Business Media B.V. 2011

\begin{abstract}
Onychomycosis is a common fungal infection of the nail but few data of mycological features in geriatric Portuguese population are yet available. The aim of this study was to perform a mycological examination and characterization of fungal nail pattern of a geriatric population from the north of Portugal clinically suspected of onychomycosis. A total of 108 patients attending the Podology Service in the Centro Hospitalar do Alto Ave (Portugal) from October 2007 to January 2009 were enrolled. All were suspected of having onychomycosis by the abnormal appearance of their nails. From these, 59.3\% were diabetic. Distal and lateral subungual onychomycosis was the more common clinical pattern followed by total dystrophic onychomycosis. In $21.3 \%$ cases, every nail in both feet had an abnormal appearance. In $86 \%$, the hallux was involved in at least one foot. Fifty samples were culture positive, and fifty-four isolates were reported regardless of the questionable pathogenicity of the infectious agent. In three cases, clinical feature of the nail, direct microscopy, and culture were consistent with
\end{abstract}

N. Dias $(\bowtie) \cdot$ C. Santos · N. Lima

(IBB) Institute for Biotechnology and Bioengineering, Centre for Biological Engineering, Universidade do Minho, Campus de Gualtar, 4710-057 Braga, Portugal e-mail: nidias@deb.uminho.pt

N. Dias · M. Portela

(CITS) Centro de Investigação em Tecnologias da Saúde, R. Central de Gandra, 4585-116 Gandra (Paredes), Portugal
Scopulariopsis infection. Fusarium spp. were identified in three cases; however, only one isolate was preceded by the observation of branching septate filaments by direct microscopy. No mixed infections with dermatophytes were reported. Trichophyton rubrum was the dermatophyte most frequently isolated (83.3\%) followed by Trichophyton interdigitale. In Portugal, onychomycosis is still viewed by general population as a cosmetic condition. Health risk is enhanced in geriatrics that only perceived the severity of their condition when experiencing further foot complications that include bacterial infection and pain.

Keywords Onychomycosis - Dermatophyte · Geriatrics

\section{Introduction}

Onychomycosis is the term generically used to describe infection of the nails due to fungi and has been reported as a major cause of nail abnormalities in developed countries [1]. The condition is worldwide in occurrence; in Europe, some populationbased studies estimated a prevalence ranging of about $3 \%$ [2,3] to 8\% [4]. Recently, the Achilles Project estimated the prevalence of onychomycosis in Europe at approximately 27\% [5]. Aging is one of the predisposing conditions to develop mycotic nails [2, 5-7], those often present chronic health problems, 
such as diabetes and poor peripheral circulation [7, 8]. In fact, in the elderly, the normal structure of the nail is altered producing changes in the color, thickness, flexibility, and shape resulting in some cases to marked dystrophic disturbances, mainly on the toenails. Since most of the functions of the nails are lost, the nail is increasingly exposed to trauma [79] and consequently to bacterial and fungal infections. Besides the effects on patient's emotional, social, and occupational functioning [10, 11], untreated onychomycosis of the toenail can lead to patient's experiencing physical impairment and pain $[12,13]$ to secondary bacterial infections and cellulitis [14]. Other toenail disorders such as trauma, onychogryphosis, lichen planus, and psoriasis may cause dystrophic or discolored nails so antifungal treatment should be exclusively reserved for those with a proved fungal infection [15]. In Portugal, treatment of this condition is often applied only based on clinical appearance of the toenail. Besides the risk of misdiagnosis and consequently incorrect treatment [16], when treating elderly patients, clinicians may be concerned about the risk of adding systemic drugs to the often long list of concomitant medications taken for comorbidities. As a result, these patients may not receive appropriate therapy. The declining health of the aging Portuguese population and the lack of published information about mycological features of onychomycosis in that highly vulnerable population lead us to carry out the mycological examination and characterization of fungal nail pattern of a geriatric population from the north of Portugal that was clinically suspected of onychomycosis. We believe that this study will help us to recognize which etiological agents are involved in toenail infection and, consequently, prescribe a suitable therapy for elderly patients.

\section{Patients and Methods}

In the period of October 2007 to January 2009, a total of 230 patients attending the Podology Service in the Centro Hospitalar do Alto Ave (Guimarãres) were clinically diagnosed of having onychomycosis. Criteria for clinical diagnosis were onycholysis, discoloration, or hyperkeratosis. Psoriatic nails, patients with eczema or lichen planus, were excluded from the study in the first place. From those, 88 patients aged below than 60 years were also excluded, and from the remaining 142 patients, 34 were excluded. Because of their prior antifungal treatment they were likely to produce negative results on mycological examination. A total of 108 patients (from 60 to 95 years) were then enrolled in this descriptive study. Informed consent was obtained. It was proposed to each subject to complete a questionnaire containing demographic and specific data, including, nail traumatism, foot hyperhidrosis, bad peripheral circulation, and predisposing diseases such as diabetes, hypertension, and cardiovascular pathologies, AIDS, tuberculosis, psoriasis, obesity, tinea pedis, or mycosis occurrence in other part of the body. The number of infected nails and great toe involvement was recorded. The type of lesion was documented and followed the classification of onychomycosis [17] as distal and lateral subungual onychomycosis (DLSO), superficial onychomycosis (SO), endonix onychomycosis (EO), proximal subungual onychomycosis (PSO), and total dystrophic onychomycosis (TDO).

\section{Mycologic Evaluation}

Nail specimens were obtained after clipping the nail and scrapping the nail bed and below the ungual plate with a sterilized scalpel. Nail material was placed on a sterile Petri dish to be sent to the laboratory. Samples were divided in three portions. Direct microscopy observation of fungal structures in fresh nail material, previously digested in a solution containing potassium hydroxide $10 \%$ and dimethylsulfoxide $40 \%$ (KOH/DMSO) (Merck), was performed with one portion. Staining with calcofluor white MR2 (CW) $0.5-1.0 \%$ (Molecular Probes) in distilled water was carried out on the second portion, and the observation was performed using an Axioskop epifluorescence microscope (Carl Zeiss). The excitation wavelength for CW stain was $346 \mathrm{~nm}$. The presence of fungal structures in stained material indicated microscopically positive specimens. Additionally, a third portion (at least ten finely scrapped nail pieces) was inoculated in Sabouraud dextrose agar (SDA-Difco) and in Dermasel agar (Difco) supplemented with cycloheximide $0.2 \%(\mathrm{w} / \mathrm{v})$ and chloramphenicol $0.025 \%$ (w/v). Dermasel agar was used in order to select dermatophytes, and the incubation was carried out at $25^{\circ} \mathrm{C}$. All cultures were 
examined twice a week for growth for up to 4 weeks before declaring them negative. Patients did not allow collecting a second sample in this study to confirm culture-based diagnosis.

\section{Isolation and Identification}

Each fungal colony growing from inoculate pieces was identified at least to the genus level, and any dermatophyte found in culture confirmed the diagnosis of onychomycosis. Identification of isolated molds and yeasts was performed taking into consideration colonial traits as texture, surface, and reverse color and/or pigmentation in malte extract agar (MEA-Oxoid) and when appropriated in potato dextrose agar (PDAOxoid). Isolation temperatures for the purpose of identification were performed at $28 \pm 2{ }^{\circ} \mathrm{C}$ for filamentous fungi and at $36^{\circ} \mathrm{C}$ for Candida optimal growth. Light microscopy observation of traits from each taxon was also performed. Nevertheless, any dermatophyte and Candida sp. found in culture were identified to the species level. For this purpose, dermatophytes were identified using macro- and micro-morphologies observations as well as yeast was presumptively identified using Cornmeal Tween80 agar for morphological identification. For these cases, matrix-assisted laser desorption/ionization time-of-flight intact cell mass spectrometry (MALDITOF ICMS) was used as confirmative test since spectra generated are characteristic for individual microbial taxon and allow the identification of isolates. The analyses were performed using an Axima LNR equipment (Kratos Analytical, Shimadzu, Manchester, UK) equipped with a nitrogen laser (337 nm). Escherichia coli DH5 $\alpha$ strain with known mass values of ribosomal proteins was used as external calibrate. For each spectrum generated, the resulting peak list, on the range between 2,000 and 20,000 $\mathrm{kDa}$, was exported to the SARAMIS ${ }^{\mathrm{TM}}$ software package (Spectral Archiving and Microbial Identification System, AnagnosTec, Postdam-Golm, Germany) in order to compare with the spectra archived on the database.

\section{Statistical Analysis}

Statistical Package for Social Studies 15.0 was used for data analysis. $P \leq 0.05$ was considered statistically significant. Binomial exact test was used to examine the distribution of a single dichotomy (gender) in a sample. Chi-square for the independence of variables was performed in order to examine proportions of two variables and their association. Mann-Whitney $U$ test was used to compare two independent groups in variables with nonparametric distribution.

\section{Results}

The major characteristics of the studied population are presented in Table 1. A total of 108 patients with a mean age of $73.92 \pm 10.50$ years were enrolled in the study. More women than men attended podologist's office $(P=0.026)$. However, when positive cases were established the proportion of women with onychomycosis was slightly higher but not significantly $(P=0.843)$ than men. About half of the patients examined declared recurrence of the infection and $50 \%$ of them referred having sometime used an antifungal treatment. Nevertheless, treatment was achieved at least 2-3 months from the time of examination.

Distal and lateral subungual onychomycosis (DLSO) was the commonest clinical pattern (59.3\%) followed by total dystrophic onychomycosis (TDO) reported in $24.1 \%$ nails, and superficial onychomycosis ( $\mathrm{SO}$ ) was found in $4.6 \%$. No cases of proximal subungual onychomycosis (PSO) or endonix onychomycosis (EO) were reported. However, 13 cases exhibited more than one type of onychomycosis on their feet, although in different toenails. From those, eight cases were clinically diagnosed as DLSO + TDO and five cases of $\mathrm{SO}+\mathrm{TDO}$, respectively. The number of involved toenails in patients suffering from TDO was significantly greater $(P<0.0001)$ than the number of involved toenails in patients suffering from other pattern of fungal infection. In our study, 59.3\% of the geriatric patients presented diabetes mellitus type II, followed by $44.4 \%$ that mentioned hypertension, $33.3 \%$ peripheral vascular problems and $32.4 \%$ cardiovascular disease. Almost fifteen percent of cases mentioned foot hyperhidrosis.

"Among 108 samples, 44 (40.7\%) were found positive by direct observation and from those only 23 were also positive for culture. A total of 37 samples in 108 samples (34.3\%) inoculated, no fungal growth 
Table 1 Demographic and clinical characteristics of the 108 patients examined
NS Not significant, $C W$

Calcofluor white; (+) positive, DLSO Distal lateral subungual onychomycosis, $S O$

Superficial onychomycosis, PSO Proximal subungual onychomycosis, $E O$ Endonix onychomycosis, $T D O$ Total dystrophic onychomycosis

a Binomial test

b Mann-Whitney $U$ test

\begin{tabular}{|c|c|c|}
\hline Parameter & $N(\%)$ & $P$ Value \\
\hline Gender & $108(100)$ & $0.026^{\mathrm{a}}$ \\
\hline Male & $42(38.9)$ & \\
\hline Female & $66(61.1)$ & \\
\hline Gender (onychomycosis positive) & & NS \\
\hline Male & 19 & $0.843^{\mathrm{a}}$ \\
\hline Female & 28 & \\
\hline Age of patients (mean \pm SD) & $73.92 \pm 10.50$ & \\
\hline \multicolumn{3}{|l|}{ Laboratorial diagnosis } \\
\hline Microscopy $(+)$ & $44(40.7)$ & \\
\hline Culture $(+)$ & $50(46.3)$ & \\
\hline Microscopy and culture $(+)$ & $23(21.3)$ & \\
\hline Microscopy and culture (-) & $37(34.3)$ & \\
\hline No of toenail involved & (median; min-max) & $<0.0001^{\mathrm{b}}$ \\
\hline No of toenail involved in TDO group & $6.00 ; 1-10$ & \\
\hline No of toenail involved in other clinical forms & $2.00 ; 1-8$ & \\
\hline Involvement of at least one great toenail & $93(86.1)$ & \\
\hline \multicolumn{3}{|l|}{ Diseases other than onychomycosis } \\
\hline Diabetes mellitus type II & $64(59.3)$ & \\
\hline Hypertension & $48(44.4)$ & \\
\hline Peripheral vascular problems & $36(33.3)$ & \\
\hline Cardiovascular disease & $35(32.4)$ & \\
\hline Foot hyperhidrosis & $16(14.8)$ & \\
\hline Obesity & $3(2.8)$ & \\
\hline Mycosis on other part of the body & $3(2.8)$ & \\
\hline Tuberculosis & $1(0.9)$ & \\
\hline Athlete's foot & $1(0.9)$ & \\
\hline AIDS & $0(0.0)$ & \\
\hline First occurrence & $51(47.2)$ & \\
\hline Pattern of fungal nail infection & 108 & \\
\hline DLSO & $64(59.3)$ & \\
\hline SO & $5(4.6)$ & \\
\hline PSO & $0(0.0)$ & \\
\hline $\mathrm{EO}$ & $0(0.0)$ & \\
\hline TDO & $26(24.1)$ & \\
\hline DLSO + TDO & $8(7.4)$ & \\
\hline $\mathrm{SO}+\mathrm{TDO}$ & $5(4.6)$ & \\
\hline
\end{tabular}

was reported in any media, neither fungal elements were observed by direct microscopy". Fifty samples $(46.3 \%)$ were grown in culture media, and fifty-four isolates were reported regardless of the questionable pathogenicity of the infectious agent (Table 2). From those, Scopulariopsis brevicaulis was found in three patients and accounted as possible etiologic agent as characteristic conidia were previously observed in fresh specimen. Furthermore, clinical appearance of the nail was consistent with Scopulariopsis infection. Fusarium spp. were identified in three cases; however, in only one case, growth in culture was preceded by observation of branching septate filaments by direct microscopy. Although basidiomycetous yeast from genus Rhodotorula was frequently found in culture, it did not accounted as presumable etiologic agent of onychomycosis. In only one case, yeast cells were observed on fresh specimen and 
Table 2 Distribution of isolates grown on culture media

\begin{tabular}{ll}
\hline Organisms & $\begin{array}{l}\text { Number of } \\
\text { isolates }\end{array}$ \\
\hline Dermatophytes & 12 \\
Trichophyton rubrum & 10 \\
Trichophyton interdigitale & 2 \\
Candida parapsilosis & 1 \\
Molds & 41 \\
Acremonium spp. & 2 \\
Alternaria alternata & 4 \\
Aspergillus spp. & 7 \\
Aspergillus versicolor & 1 \\
Cladosporium spp. & 5 \\
Curvularia spp. & 5 \\
Fusarium spp. & 3 \\
Penicillium spp. & 7 \\
Phialophora spp. & 1 \\
Scedosporium (Pseudallasheria) & 1 \\
Scopulariopsis brevicaulis & 3 \\
Stemphylium spp. & 1 \\
Ulocladium spp. & \\
Total & \\
\hline
\end{tabular}

thereafter Candida parapsilosis was grown in culture. Clinical file revealed that the female patient suffered from repeated urinary tract infection episodes and consequently took antibiotics frequently. Furthermore, the patient was diabetic. Antropophilic species of Trichophyton rubrum and Trichophyton interdigitale were identified in a total of 12 dermatophytes samples accounting for 83.3 and $16.7 \%$, respectively. Distribution of fungi isolated from each pattern of fungal nail infection was represented on Fig. 1. Dermatophytes and nondermatophytes were found as causative agents of onychomycosis in DLSO and TDO, but not in SO pattern. Candida parapsilosis was found from a patient clinically suspected of DLSO.

\section{Discussion}

Factors that can contribute to the increased prevalence of onychomycosis in the geriatric population include a reduced rate of nail growth and a greater frequency of trauma with the consequence of a more propitious opportunity for causing disease in the nail bed and plate [18]. Total dystrophic onychomycosis

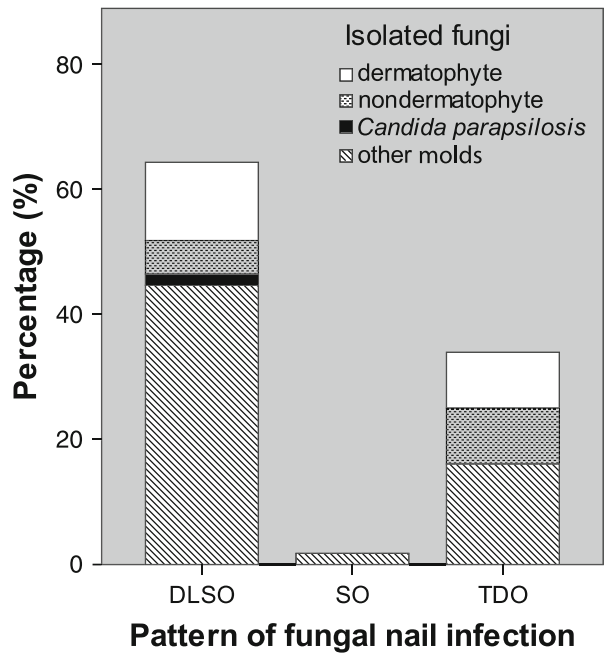

Fig. 1 Distribution of fungi isolated from each pattern of fungal nail infection. $P S O$ (proximal subungual onychomycosis) and $E O$ (endonix onychomycosis) were not included as each one accounted for a percentage of zero fungi for these patterns

(TDO) has been reported as the most destructive clinical form representing the end result of the evolution of any of the other three clinical forms [8]. In our study, this clinical presentation occurred in 39 cases, suggesting an advanced stage of the disease. In addition, the high number of involved toenails found on TDO cases also suggested a long-time exposure to pathogenic agents before sought for medical care. Hallux toenail has been reported [18] as the most affected nail due to direct trauma predisposition. The involvement of at least one hallux was found in 93 of the 108 patients that represent $86.1 \%$ of the sampling population, still none of the cases mentioned suffering from a recent or ancient nail trauma. From our clinical experience, most of nail fungal infections are the result of old (more than 5 years) trauma instead of recent ones. Our results are in agreement with Szepietowski et al. [19] that performed a large study in Poland and concluded that onychomycosis in geriatric populations were characterized by a higher number of involved toenails, a longer duration of the disease, and significantly more advanced lesions. The low-income level in this particular group, most being retired, along with high costs related to foot care may explain the decision of not to treat the disease in the early stages.

In geriatrics, the development of chronic health problems, such as poor peripheral vascular 
circulation or diseases like diabetes or cardiovascular complications is common, which may predisposes to fungal nail infections $[7,20]$. In our study, the same health conditions were mentioned by a high percentage of patients.

Dermatophytes were found in only 12 over the 54 isolates; however, arthroconidia and hyphae of regular width were observed in 44 preparations. This result suggests that about $50 \%$ of samples presumably infected by dermatophytes did not grow on the culture medium. Negative growth culture from microscopically positive specimen is a well-known phenomenon. Sampling of nail material from patients who underwent antifungal treatment reduces culture rates [21]. In this study, this occurrence was prevented since an a priori exclusion was performed on patients which antifungal treatment was not achieved at least 2-3 months from the time of examination. However, we can not rule out the presence of nonviable or scarce fungal fragments in specimen resulting in $\mathrm{KOH}$ positive but culturenegative sample. Despite the high percentage of false-negative culture rate, mycological examination remains the currently preferred diagnostic method [22]. Identification of the pathogen must be performed to optimize treatment [23]. Trichophyton rubrum was the most common isolated dermatophyte in this study, as it was expected, once this antropophilic species is the most common dermatophyte in skin and nail infections since last 55 years [24-27]. In Portugal, Valdigen et al. [28], which underwent a twenty-year retrospective study in the North of Portugal (Braga), reported also a changing in dermatophyte predominance pattern, being $T$. rubrum the most isolated dermatophyte in superficial fungal infections.

Besides the controversy on the clinical significance of nondermatophyte (NDF) molds as etiologic agents of onychomycosis, it was not possible in this study to apply with accuracy the criteria for nondermatophyte outgrowth for nail infection established by English [29]. Ideally, a second sample should be examined to confirm the causative agent $[20,22,30]$ based on the premise that a contaminant rarely is found consistently in two consecutive samples [31]. The authors admitted that failure to resample the patients is a limitation of this study. In fact, patients were not available to take a second sample and it could contribute for the high percentage of molds found in this study.

\section{Conclusion}

Chronic onychomycosis may increase the costs of health care. In this study, a high percentage of patients presenting other diseases besides fungal infection alert from the fact that care should be taken by clinicians in geriatric individuals. Direct examination and culture are fundamental tools to give the correct diagnosis before starting any treatment, and sampling should be routinely performed. Larger studies should be undertaken leading to the definitive identification of NDF infections in nails through the isolation of the agent in successive specimens from the infected regions.

\section{References}

1. Midgley G, Moore MK. Onychomycosis. Rev Iberoam Micol. 1998;15:113-7.

2. Roberts DT. Prevalence of dermatophyte onychomycosis in the United Kingdom: results of an omnibus survey. Br J Dermatol. 1992;126((S39)):23-7.

3. Perea S, Ramos MJ, Garau M, Gonzalez A, Noriega AR, del Palacio A. Prevalence and risk factors of tinea unguium and tinea pedis in the general population in Spain. J Clin Microbiol. 2000;38:3226-30.

4. Sigurgeirsson B, Steingrímsson O, Sveinsdóttir S. Prevalence of onychomycosis in Iceland: a population-based study. Acta Derm Venereol. 2002;82:467-9.

5. Burzykowski T, Molenberghs G, Abeck D, Haneke E, Hay R, Katsambas A, et al. High prevalence of foot diseases in Europe: results of the Achilles Project. Mycoses. 2003;46:496-500.

6. Elewski BE. Onychomycosis: pathogenesis, diagnosis and management. Clin Microbiol Rev. 1998;11:415-29.

7. Tosti A, Hay R, Arenas-Guzmán R. Patients at risk of onychomycosis: risk factor identification and active prevention. Eur Acad Dermatol Venereol. 2005;19((S1)):13-6.

8. Jaffe R. Onychomycosis: recognition, diagnosis, and management. Arch Fam Med. 1998;7:587-92.

9. Torres-Rodríguez JM, López-Jodra O. Epidemiology of nail infection due to keratinophilic fungi. Rev Iberoam Micol. 2000;17:1-12.

10. Drake LA, Scher RK, Smith EB, Faich GA, Smith SL, Hong JJ, et al. Effect of onychomycosis on quality of life. J Am Acad Dermatol. 1998;38:702-4.

11. Drake LA, Patrick DL, Fleckman P, André J, Baran R, Haneke E, et al. The impact of onychomycosis on quality of life: development of an international onychomycosis- 
specific questionnaire to measure patient quality of life. J Am Acad Dermatol. 1999;41:189-96.

12. Lubeck DP. Measuring health-related quality of life in onychomycosis. J Am Acad Dermatol. 1998;38((Part3)): S64-6.

13. Mayser P, Freund V, Budihardja D. Toenail onychomycosis in diabetic patients: issues and management. Am J Clin Dermatol. 2009;10:211-20.

14. Rich P. Onychomycosis and tinea pedis in patients with diabetes. J Am Acad Dermatol. 2000;43:S130-4.

15. Scher RK, Tavakkol A, Sigurgeirsson B, Hay RJ, Joseph WS, Tosti A, et al. Onichomycosis: diagnosis and definition of cure. J Am Acad Dermatol. 2007;56:939-44.

16. de Berker D. Fungal nail disease. N Engl J Med. 2009;360:2108-16.

17. Baran R, Hay RJ, Tosti A, Haneke E. A new classification of onychomycosis. Br J Dermatol. 1998;139:567-77.

18. de Araújo AJG, Bastos OM, Souza MAJ, Oliveira JC. Ocorrência de onicomicose em pacientes atendidos em consultórios dermatológicos da cidade do Rio de Janeiro, Brasil. Ann Bras Dermatol. 2003;78:299-308.

19. Szepietowski JC, Reich A, Garlowska E, Kulig M, Baran E. Factors influencing coexistence of toenail onychomycosis with tinea pedis and other dermatomycoses. Arch Dermatol. 2006;142:1279-84.

20. Saunte DM, Holgersen JB, Haedersdal M, Strauss G, Bitsch M, Svendsen OL, et al. Prevalence of toe nail onychomycosis in diabetic patients. Acta Derm Venereol. 2006;86:425-8.

21. Robert R, Pihet M. Conventional methods for the diagnosis of dermatophytosis. Mycopathologia. 2008;166:295-306.
22. Feuillade de Chauvin M. New diagnostic techniques. Eur Acad Dermatol Venereol. 2005;19((S1)):20-4.

23. Hay R. Literature review. Eur Acad Dermatol Venereol. 2005;19((S1)):1-7.

24. Roberts DT, Taylor WD, Boyle J. Guidelines for treatment of onychomycosis. Br J Dermatol. 2003;148:402-10.

25. Effendy I, Lecha M, Feuilhade de Chauvin M, Di Chiacchio $\mathrm{N}$, Baran R. Epidemiology and clinical classification of onychomycosis. J Eur Acad Dermatol Venereol. 2005; 19((S1)):8-12.

26. Seebacher C, Brasch J, Abeck D, Cornely O, Effendy I, Ginter-Hanselmayer G, et al. Onychomychosis. Mycoses. 2007;50:321-7.

27. Seebacher C, Bouchara JP, Mignon B. Updates on the epidemiology of dermatophyte infections. Mycopathologia. 2008;166:335-52.

28. Valdigen GL, Pereira T, Macedo C, Duarte ML, Oliveira P, Ludovico P, et al. A twenty-year survey of dermatophytosis in Braga, Portugal. Int J Dermatol. 2006;45:822-7.

29. English MP. Nails and fungi. Br J Dermatol. 1976;94: 697-701.

30. Arrese JE, Piérard-Franchimont C, Piérard GE. Facing up to the diagnostic uncertainty and management of onychomycoses. Int J Dermatol. 1999;38((S2)):1-6.

31. Summerbell RC, Cooper E, Bunn U, Jamieson F, Gupta AK. Onychomycosis: a critical study of techniques and criteria for confirming the etiologic significance of nondermatophytes. Med Mycol. 2005;43:39-59. 